\title{
Kieferorthopädische Behandlungselemente aus pseudoelastischen Nickel-Titan-Memory-Legierungen - Finite Elemente Modellierung, experimentelle Verifizierung und klinischer Einsatz \\ C. Bourauel', D. Drescher ${ }^{1}$, L.-P. Nolte ${ }^{2}$, D. Kobe ${ }^{1}$ \\ ${ }^{1}$ Poliklinik für Kieferorthopädie, Universität Bonn \\ 2 Maurice E. Müller Institut für Biomechanik, Universităt Bern
}

\section{Einleitung}

Im Verlauf einer kieferorthopädischen Behandlung können Zähne oder Zahngruppen über mehrere Millimeter durch den Kieferknochen bewegt werden. Eine zielgerichtete Planung der Behandlung erfordert den Einsatz von Behandlungselementen mit definiertem mechanischem Verhalten und möglichst konstantem Kraftsystem. Die Entwicklung derartiger aktiver Elemente wurde in den letzten Jahren durch die Einführung von Nickel-Titan-Formgedächtnis-Legierungen in die Kieferorthopädie ermöglicht $(1,5)$.

Nickel-Titan-Legierungen (NiTi) weisen zwei außergewöhnliche mechanische Eigenschaften auf: den Formgedächtnis- oder Memory-Effekt und die Pseudoelastizităt. Wăhrend die Anwendung des Formgedächtnis-Effektes verschiedene technische Probleme aufwirft, werden in der Kieferorthopädie Drähte aus pseudoelastischen NiTi-Legierungen zur Korrektur von Zahnfehlstellungen bereits in großem Umfang eingesetzt. Das Spannungs/Dehnungs-Diagramm eines ein-axialen Zugversuchs an einer NiTiLegierung zeigt eine extreme Nichtlinearităt, die anhand von Abb. 1 erklärt werden soll:

bis zu einer relativen Dehnung von ca. 1,5\% (Punkt A) verhält sich das Material linear. Es schließt sich ein nichtlinearer Bereich an, auf den das sogenannte pseudoelastische Plateau folgt. Dieses ist charakterisiert durch einen weiten Dehnungsbereich zwischen ca. $1,5 \%$ und $6,0 \%$ Dehnung (zwischen den Punkten $A$ und $B$ ), in dem die Spannung nahezu konstant bleibt. Anschließend erfolgt ein linearer Anstieg der Spannung mit der Dehnung bis zur Elastizitätsgrenze $(\mathrm{M})$, die bei einigen NiTi-Legierungen $8 \%$ Dehnung

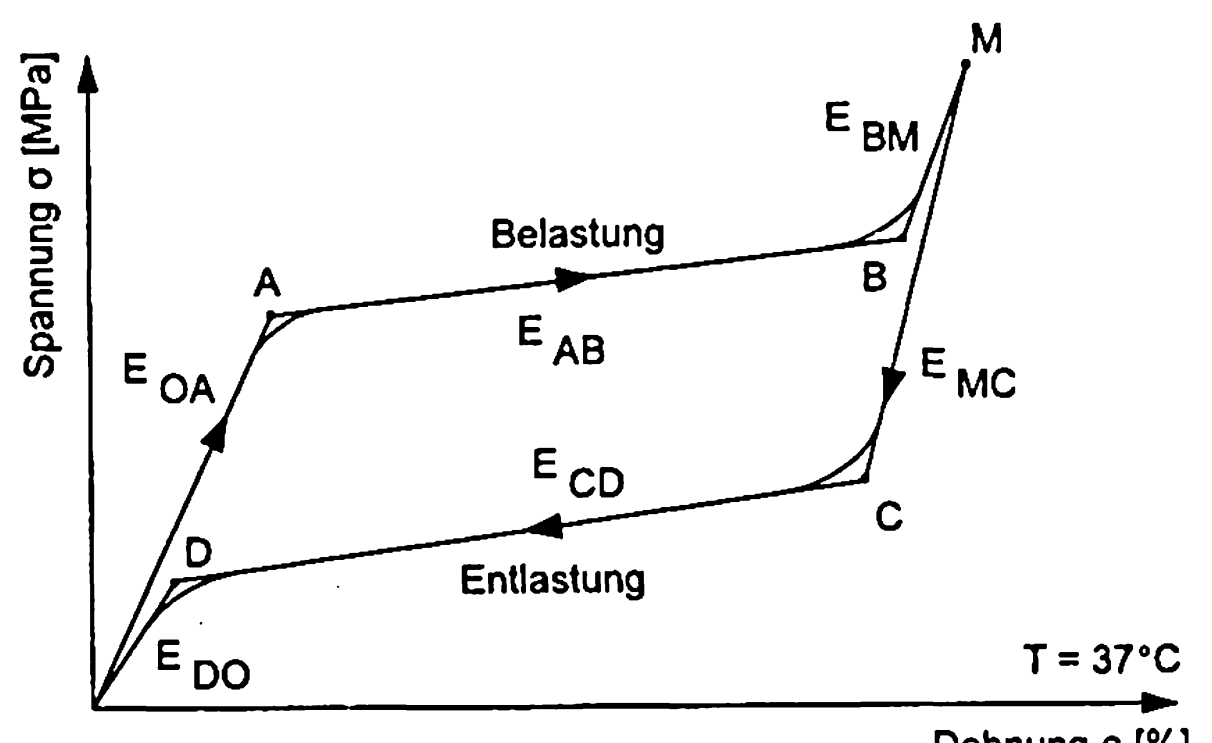

Abb. 1: Schematisches Spannungs/Dehnungs-Diagramm einer pseudoelastischen Legierung erreichen kann. Bei Entlastung des Materials zeigt sich wiederum ein ähnliches Verhalten. Da die Elastizitätsparameter gegenüber der Belastung jedoch leicht geändert sind, zeigt sich eine breite Hysterese, die für pseudoelastische NiTi-Legierungen typisch ist. Eine Übertragung dieser Legierungseigenschaften auf kieferorthopädische Behandlungselemente läßt ein außergewöhnliches mechanisches Verhalten erwarten.

\section{Methode}

Für die Entwicklung pseudoelastischer Behandlungselemente aus NiTi-Legierungen wurde ein rumerisches Modell auf der Basis der Methode der Finiten Elemente entwickelt. Das Modell wurde in ein bestehendes FEM-Paket implementiert (8) und in eine speziell auf den kieferorthopädischen Anwendungsfall ausgerichtete Benutzeroberfläche integriert. Sowohl das zweidimensionale pseudoelastische Finite Element als auch die Benutzeroberfläche SOLF (Simulation Orthodontischer Loops mit FEM) wurden bereits an anderer Stelle beschrieben $(3,9)$. Das Programmsystem erlaubt das interaktive Design beliebiger ebener Federgeometrien, die Berechnung des Last Deformations-Verhaltens und die Darstellung der Ergebnisse auf dem Bildschirm.

Zur Verifizierung der numerischen Ergebnisse wurden die berechneten Federgeometrien aus kieferorthopädischen NiTi-Drähten mit dem Querschnitt $0,41 \mathrm{~mm} \times$ $0,56 \mathrm{~mm}$ hergestellt und in dem biomechanischen Meßaufbau OMSS (Orthodontisches Meß- und Simulations-System) vermessen. Der Aufbau des OMSS ist in (2) und (6) beschrieben. Das Meßsystem

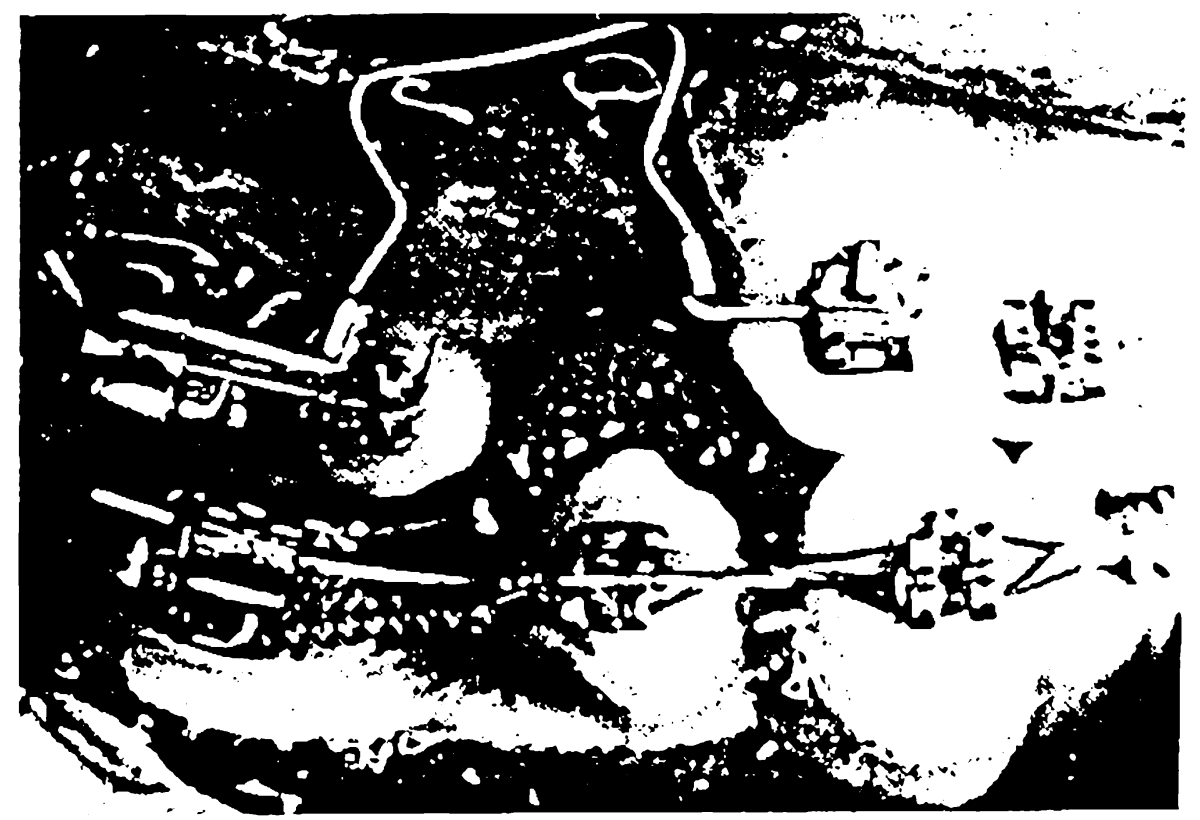

Abb. 2: Einsatz einer pseudoelastischen T-Feder 
kann an zwei Meßstellen jeweils drei Kräfte und drei Drehmomente mit einer Auflosung von $0,01 \mathrm{~N}$ bzw. $0,1 \mathrm{Nmm}$ aufnehmen und jede beliebige Starrkörperbewegung im Raum ausführen. Somit ist eine anwendungsorientierte Kalibrierung von kieferorthopădischen Behandlungselementen möglich.

Mit diesen numerischen und experimentellen Methoden wurden Behandlungselemente für die Eckzahnretraktion (4) und für die Aufrichtung gekippter Molaren (7) entwickelt. Abb. 2 zeigt als Beispiel die klinische Anwendung einer NiTi-T-Feder zur Eckzahnretraktion.

\section{Ergebnisse}

Abb. 3 zeigt den Vergleich der berechneten und gemessenen Hysteresekurve für die T-Feder aus Abb. 2. Die Feder hat eine Höhe von $10 \mathrm{~mm}$ und eine Länge des T-Segments von ebenfalls $10 \mathrm{~mm}$. Die pseudoelastischen Parameter der verwendeten NiTi-Legierung (GAC-Sentalloy ${ }^{\mathrm{tm}}$ ) sind im folgenden zusammengefaßt, die Definitionen entsprechen der Abb. 1: $\epsilon_{A}: 0,73 \%, \quad \epsilon_{B}: 5,28 \%$

$E_{O A}: 57,5 M P a, E_{A B}: 0,24 M P a, E_{B M}: 24,1 M P a$, $\epsilon_{\mathrm{C}}: 0,28 \%$,

$E_{D 0}: 45,1 \mathrm{MPa}, E_{C D}: 1,3 \mathrm{MPa}, \quad E_{M C}: 25,4 \mathrm{MPa}$ Deutlich sind die pseudoelastischen Plateaus sowohl bei Be- als auch bei Entlastung zu erkennen. Die Kräfte auf den Plateaus liegen zwischen 1,5N und 2,0N (Belastung) bzw. zwischen 1,0N und 0,4N (Belastung). Da für die kieferorthopädische Behandlung der Entlastungsast relevant ist, sollte während der Bewegung eine Kraft von maximal 1,0N auf den Zahn einwirken. Dieser Wert ist hervorragend für die gewählte Anwendung geeignet. Der Vergleich der experimentellen und numerischen Ergebnisse zeigt eine Abweichung auf dem Entlastungsast von ca. 0,4N. Diese kann durch eine martensitische Deformation des NiTi-Drahtes erklärt werden, die bei Erwärmung wieder zurückgeht. Im übrigen liegt die Übereinstimmung von berechneten und gemessenen Daten bei ca. $10 \%$.

\section{Diskussion}

Die vorgestellten Ergebnisse zeigen, daß aus kieferorthopădischen NiTi-Drähten Behandlungselemente mit ungewöhnlichen mechanischen Eigenschaften

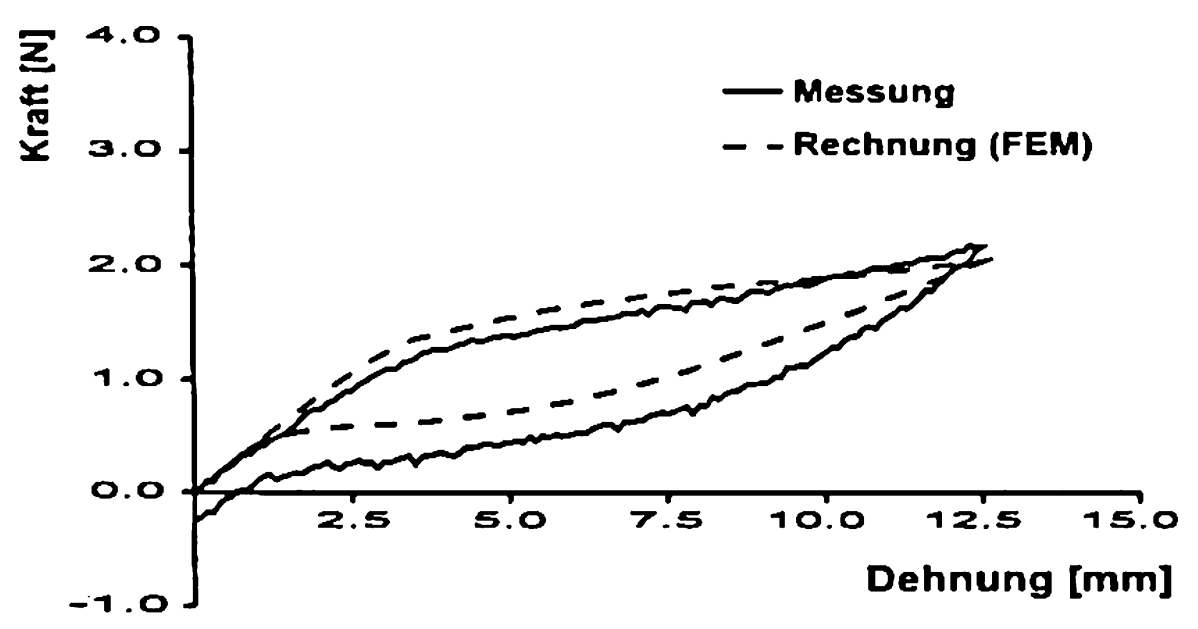

Abb. 4: Vergleich der gemessenen und berechneten Last/Auslenkungs-Kurve einer NiTi-T-Feder

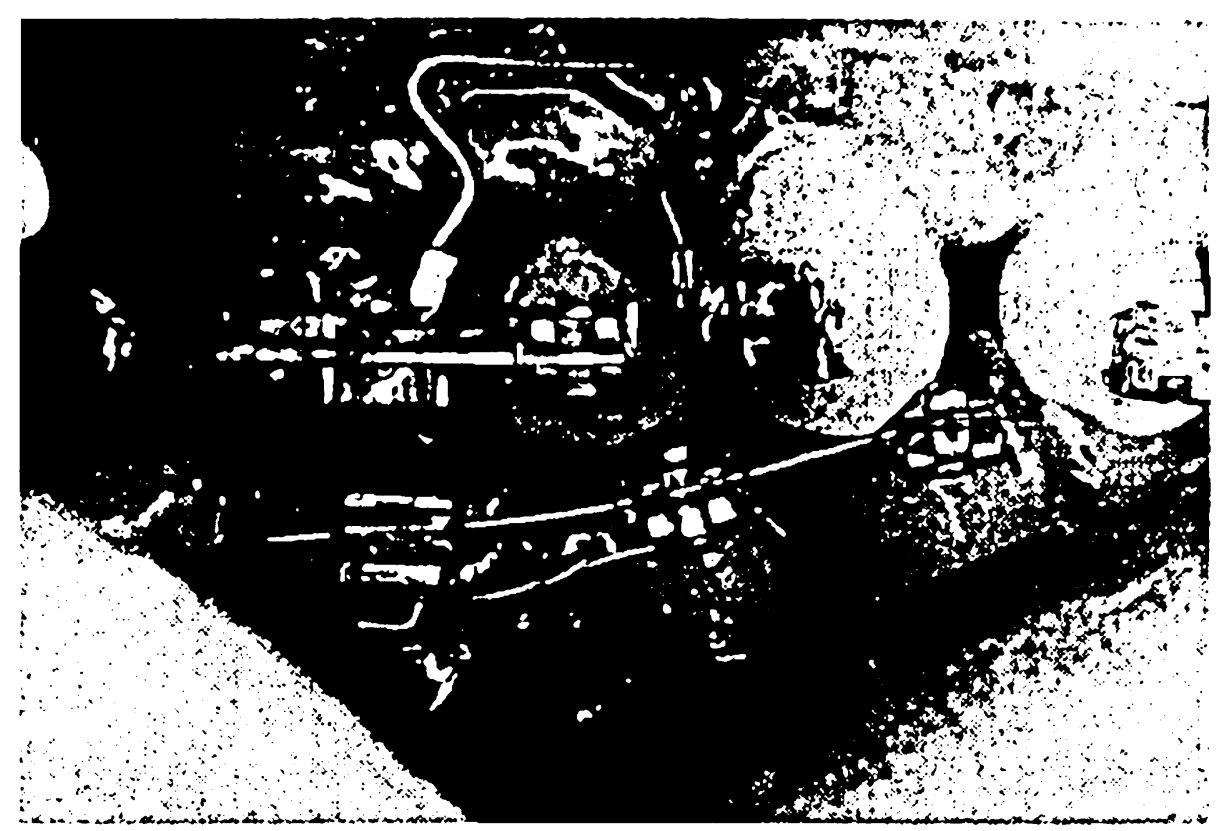

Abb.4: Abschlußsituation einer Eckzahnretraktion

hergestellt werden können. Die klinische Anwendung (vergl. Abb. 2 und 4) zeigt, daß mit pseudoelastischen Federn nebenwirkungsarme, zielgerichtete Zahnbewegungen zu erzielen sind.

\section{Literatur}

(1) Andreasen, G.F. und Brady, P.R.: An evaluation of 55 cobalt substituted nitinol wire for use in orthodontics. J. American Dental Association 82, 1373-1375 (1971)

(2) Bourauel, C., Drescher, D. und Thier, M.: An experimental apparatus for the simulation of three-dimensional movements in orthodontics. J. Biomedical Engineering 14, 371-378 (1992)

(3) Bourauel, C., Nolte, L.P. und Drescher, D.: Numerische Untersuchung kieferorthopädischer Behandlungselemente aus pseudoelastischen NiTi-Legierungen. Biomedizinische Technik 37, 46-53 (1992)

(4) Bourauel, C., Drescher, D. und Nolte, L.P.: Computergestützte Entwicklung kieferorthopädischer Behandlungselemente aus NiTi-Memory-Legierungen am Beispiel einer pseudoelastischen Retraktionsfeder. Fortschritte der Kieferorthopädie 53, 45-56 (1993)

(5) Buehler, W.J., Gilfrich, J.V. und Wiley, R.C.: Effect of low-temperature phase changes on the mechanical properties of alloys near composition of TiNi. J. Applied Physics 34, 1475-1484 (1963)

(6) Drescher, D., Bourauel, C. und Thier, M.: Application of the orthodontic measurement and simulation system (OMSS) in orthodontics. European J. of Orthodontics 13, 169-178 (1991)

(7) Drescher, D., Bourauel, C. und Thier, M.: Eine pseudoelastische NiTi-Aufrichtefeder für Molaren Entwurf, biomechanische Prüfung und klinische Anwendung. Fortschritte der Kieferorthopädie 53, 286296 (1992)

(8) Hartmann, B., Schrader, K.H. und Winkel, G.: MESY - Ein Programmsystem zur Untersuchung von Tragwerken. KIB-Berichte 22, Vulkan-Verlag Essen 1975

(9) Nolte, L.-P., Bourauel, C. und Drescher, D.: Orthodontic Appliances Made of Superelastic NiTi Alloy Mathematical Modelling and Experimental Validation. Clinical Biomechanics (z. Ver. einger.) 